\title{
Lidil
}

Revue de linguistique et de didactique des langues

$59 \mid 2019$

Prononcer les langues : variations, émotions, médiations

\section{Jean-Michel Adam, Le paragraphe : entre phrases et} texte

Paris, Armand Colin, 2018, 224 p.

\section{Julie Sorba}

\section{OpenEdition \\ Journals}

Édition électronique

URL : http://journals.openedition.org/lidil/6170

DOI : $10.4000 /$ lidil.6170

ISSN : $1960-6052$

\section{Éditeur}

UGA Éditions/Université Grenoble Alpes

\section{Édition imprimée}

ISBN : 978-2-37747-090-7

ISSN : $1146-6480$

\section{Référence électronique}

Julie Sorba, « Jean-Michel Adam, Le paragraphe : entre phrases et texte», Lidil [En ligne], 59 | 2019, mis en ligne le 01 mai 2019, consulté le 24 septembre 2020. URL : http://journals.openedition.org/lidil/ 6170 ; DOI : https://doi.org/10.4000/lidil.6170

Ce document a été généré automatiquement le 24 septembre 2020

(C) Lidil 


\section{Jean-Michel Adam, Le paragraphe: entre phrases et texte}

Paris, Armand Colin, 2018, 224 p.

Julie Sorba

\section{RÉFÉRENCE}

Jean-Michel Adam, Le paragraphe : entre phrases et texte, Paris, Armand Colin, 2018, 224 p.

1 Placé sous les auspices d'Henri Mitterand, présenté comme un précurseur dans la réflexion linguistique sur la place du paragraphe, l'ouvrage de Jean-Michel Adam s'articule en deux parties. La première présente les propositions de l'auteur pour remédier à « la sous-théorisation endémique du concept de paragraphe» (p. 205) et la seconde met à l'épreuve celles-ci au sein de différentes activités discursives. L'auteur propose ainsi une réflexion sur les pratiques de segmentation des textes et leur rôle dans la construction du sens, au fil du temps, dans les genres médiatiques (le fait divers) et publicitaires, mais aussi dans les genres littéraires du conte (des premières éditions de La Belle au bois dormant au XVII siècle aux traductions contemporaines du Petit Chaperon rouge), du poème en prose (Baudelaire) et du roman (Camus, Butor, Simon). Ses analyses révèlent une forte disparité des pratiques non seulement dans le temps, mais aussi à la même époque chez des auteurs et des éditeurs différents (notamment pour le conte Riquet à la houppe).

2 J.-M. Adam part d'un constat paradoxal: d'une part, le paragraphe est considérée comme une unité de discours centrale dans les domaines de l'enseignement du français et de la didactique de l'écrit ("une évidence», p. 9) et, d'autre part, peu d'études théoriques lui ont été consacrées jusqu'à présent («un objet flou», p. 9). Depuis les années 1960, la recherche anglo-saxonne en psychologie cognitive a certes reconnu le paragraphe comme une "réalité psychologique» (p.11) et de nombreuses expérimentations ont révélé que «la segmentation typographique de blocs textuels d'énoncés en paragraphes vise à programmer la lecture en donnant des instructions de 
cycles ou boucles de traitement d'empans textuels successifs»(p.11). Néanmoins, comme le souligne l'auteur à juste titre, les linguistes ont tardé à s'intéresser à cet objet alors qu'il représente "l'unité idéale d'observation des phénomènes interphrastiques » (p.12). Cette réticence peut s'expliquer par l'extrême diversité des paragraphes et la difficulté consécutive à en dresser une typologie univoque. Tout en se positionnant différemment du projet de grammaire du paragraphe de Longacre (1980), l'auteur conserve néanmoins un élément de définition proposé par ce dernier pour lequel le paragraphe représente "un niveau de composition situé entre la microsegmentation (phrastique) et la macrosegmentation (textuelle)»(p.13). L'exploration de ce palier mésotextuel de structuration du texte est précisément l'objet du présent ouvrage et le cadre dans lequel s'inscrit cette réflexion est celui de la linguistique du discours.

3 La première partie de l'ouvrage présente les choix théoriques de l'auteur en les situant dans leur contexte et en posant leurs enjeux. J.-M. Adam propose de ranger l'alinéa et le paragraphe dans une catégorie qu'il rebaptise "ponctuation transphrastique » (p. 26) en retenant finalement le classement des ponctuants proposés par C. Tournier (1980). Après avoir présenté le flottement définitionnel concernant paragraphe et alinéa, il s'inscrit dans la lignée de H. Mitterand (1985) en faisant du paragraphe "une unité textuelle transphrastique» et de l'alinéa «le signe majeur de ponctuation du paragraphe » (p. 34). À la suite de M. Charolles (1988), J.-M. Adam souligne également le rôle essentiel joué par les paragraphes dans « la gestion pragmatique des informations textuelles» (p.44): la segmentation en paragraphe est faite pour guider l'interprétation du texte.

4 Par ailleurs, tout en reconnaissant le caractère novateur de l'école anglo-saxonne de la tagmémique dans la théorisation sur le paragraphe depuis les années 1950, J.-M. Adam juge que la distinction opérée par celle-ci entre paragraphe graphique et paragraphe sémantique doit être dépassée, car « un paragraphe graphique est toujours une unité de sens rendue vi-lisible et donnée à interpréter » (p. 65). En effet, les paragraphes sont les lieux à l'échelle desquels se déploie le phénomène isotopique qui assure les opérations de liage textuel. Ainsi, l'auteur intègre le paragraphe à sa conception du texte en réunissant les deux aspects artificiellement séparés par la tagmémique: "Le paragraphe est définissable comme un SEGMENT GRAPHIQUE MÉSOTEXTUEL constitué d'un nombre indéterminé de phrases graphiques et donc d'un nombre tout aussi indéterminé de propositions. Ces suites de propositions sont, par étapes ou boucles de traitement, regroupées en paquets de propositions formant des unités sémantiques de plus haut rang de complexité : les macropropositions [MP].» (p. 94-95) Celles-ci se réalisent au sein de séquences (descriptives, narratives, argumentatives, explicatives, etc.) qui constituent une catégorie de textualisation participant à l'organisation du discours. En effet, à l'instar de D. Maingueneau (2014), J.-M. Adam (2017) postule une double organisation du discours à la fois par les genres et par un ensemble de règles transversales aux genres (dont les séquences font partie). Il réaffirme ainsi le caractère central de cette seconde catégorie: «Les modes prégénériques préformatés d'empaquetage de suites de propositions dans les MP liées formant des types de séquences sont l'objet d'un apprentissage parallèle à celui de la langue. » (p. 98) Comme ces séquences sont le plus souvent mélangées les unes aux autres dans les textes, J.-M. Adam rappelle que le plan de texte est le facteur unifiant et obligatoire des structures compositionnelles. Sur ce modèle, il développe in fine le concept de plan de paragraphe dont il reconnait les réalisations extrêmement variées dans les deux exemples qu'il 
analyse (un texte du genre encyclopédique et didactique intitulé La forêt équatoriale et le poème en prose La belle Dorothée de Baudelaire).

5 Au terme de cette démonstration, il apparait clairement que le paragraphe est « une unité pleinement linguistique»(p.201) qui mérite toute sa place au sein de la linguistique textuelle. J.-M. Adam conclut en affirmant la complémentarité de l'alinéa, "une méso-unité ponctuationnelle de segmentation», et du paragraphe, « une mésounité de sens»(p. 202). Il confirme enfin la nécessité d'intégrer la question de la segmentation graphique des textes dans une théorie des paliers de structuration qui constitue, selon lui, l'objet principal de la linguistique textuelle.

\section{AUTEURS}

JULIE SORBA

LIDILEM, Université Grenoble Alpes 